Annals of Pure and Applied Mathematics

Vol. 15, No. 2, 2017, 253-269

ISSN: 2279-087X (P), 2279-0888(online)

Published on 11 December 2017

www.researchmathsci.org

DOI: http://dx.doi.org/10.22457/apam.v15n2a12

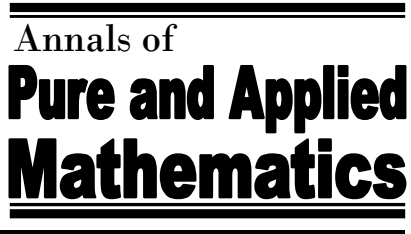

\title{
Bipolar Anti L-Fuzzy Sub $\ell$-HX Group and its Lower Level Sub $\ell$-HX Groups
}

\author{
R. Muthuraj ${ }^{1}$ and G. Santha Meena ${ }^{2}$ \\ ${ }^{1}$ PG \& Research Department of Mathematics, H.H. The Rajah's College \\ Pudukkottai - 622 001, Tamilnadu, India. rmr1973@yahoo.co.in \\ ${ }^{2}$ Department of Mathematics, PSNA College of Engineering and Technology \\ Dindigul - 624 622, Tamilnadu, India. g.santhameena@gmail.com
}

\section{Received 18 October 2017; accepted 10 December 2017}

\begin{abstract}
In this paper, we discussed some properties of bipolar anti L - fuzzy sub $\ell$ - HX group of a $\ell$ - HX group. The purpose of this study is to implement the fuzzy set theory and graph theory in bipolar anti L - fuzzy sub $\ell$ - HX group. Characterizations of lower level subsets of a bipolar anti L - fuzzy sub $\ell$ - HX group are given. We also discussed the relation between a bipolar anti L - fuzzy sub $\ell$ - HX group and its lower level sub $\ell$ HX groups and investigate the conditions under which a given sub $\ell-$ HX group has a properly inclusive chain of sub $\ell-\mathrm{HX}$ groups. In particular, we formulate how to structure a bipolar anti L - fuzzy sub $\ell$ - HX group by a given chain of sub $\ell$ - HX groups. We also establish the relation between bipolar L - fuzzy sub $\ell$ - HX group and bipolar anti L - fuzzy sub $\ell$ - HX group.
\end{abstract}

Keywords: Bipolar L - fuzzy $\ell$ - HX group, Bipolar anti L - fuzzy $\ell$ - HX group, lower level subset, lower level sub $\ell$-HX group.

AMS Mathematics Subject Classification (2010): 20N25, 03E72, 03G25

\section{Introduction}

The concept of fuzzy sets was initiated by Zadeh [14]. Then it has become a vigorous area of research in engineering, medical science, social science, graph theory etc. Rosenfeld [11] gave the idea of fuzzy subgroups. In fuzzy sets the membership degree of elements range over the interval $[0,1]$. The membership degree expresses the degree of belongingness of elements to a fuzzy set. The membership degree 1 indicates that an element completely belongs to its corresponding fuzzy set and membership degree 0 indicates that an element does not belong to fuzzy set. The membership degrees on the interval $(0,1)$ indicate the partial membership to the fuzzy set. Sometimes, the membership degree means the satisfaction degree of elements to some property or constraint corresponding to a fuzzy set. Hongxing [4] introduced the concept of HX group and the authors Chengzhong, Honghai, Hongxing [5] introduced the concept of fuzzy HX group. The author Zhang [15] commenced the concept of bipolar fuzzy sets as a generalization of fuzzy sets in 1994. Lee [3] introduced Bipolar-valued fuzzy sets and their operations. In case of Bipolar-valued fuzzy sets membership degree range is enlarged from the interval $[0,1]$ to $[-1,1]$. In a bipolar-valued fuzzy set, the membership 


\section{R. Muthuraj and G. Santha Meena}

degree 0 means that the elements are irrelevant to the corresponding property, the membership degree $(0,1]$ indicates that elements somewhat satisfy the property and the membership degree $[-1,0)$ indicates that elements somewhat satisfy the implicit counterproperty. Satya Saibaba [12] initiated the study of L - fuzzy lattice ordered groups and introduced the notions of L - fuzzy sub $\ell$ - HX group. Goguen [2] replaced the valuation set $[0,1]$ by means of a complete lattice in an attempt to make a generalized study of fuzzy set theory by studying L - Fuzzy sets. Muthuraj and Sridharan [9] introduced Bipolar Anti Fuzzy HX Group and its Lower Level sub HX groups. Sunderrajan and Senthilkumar [13] introduced Anti L-fuzzy sub $\ell$ group and its lower level sub $\ell$ groups.

\section{Preliminaries}

In this section, we site the fundamental definitions that will be used in the sequel. Throughout this paper, $\mathrm{G}=(\mathrm{G},$.$) is a group, e is the identity element of \mathrm{G}$, and $x y$, we mean $\mathrm{x} \cdot \mathrm{y}$.

Definition 2.1. A Bipolar L - fuzzy set $\mu$ in $G$ is a bipolar $L$ - fuzzy subgroup of $G$ if for all $\mathrm{x}, \mathrm{y} \in \mathrm{G}$.
i) $\quad \mu^{+}(\mathrm{xy}) \geq \mu^{+}(\mathrm{x}) \wedge \mu^{+}(\mathrm{y})$
ii) $\quad \mu^{-}(\mathrm{xy}) \leq \mu^{-}(\mathrm{x}) \vee \mu^{-}(\mathrm{y})$
iii) $\quad \mu^{+}\left(\mathrm{x}^{-1}\right)=\mu^{+}(\mathrm{x})$
iv) $\quad \mu^{-}\left(x^{-1}\right)=\mu^{-}(x)$.

Definition 2.2. A bipolar anti L - fuzzy set $\mu$ in $\mathrm{G}$ is a bipolar anti L - fuzzy subgroup of $\mathrm{G}$ if for all $\mathrm{x}, \mathrm{y} \in \mathrm{G}$.
i) $\quad \mu^{+}(x y) \leq \mu^{+}(x) \vee \mu^{+}(y)$
ii) $\quad \mu^{-}(\mathrm{xy}) \geq \mu^{-}(\mathrm{x}) \wedge \mu^{-}(\mathrm{y})$
iii) $\quad \mu^{+}\left(x^{-1}\right)=\mu^{+}(x)$
iv) $\quad \mu^{-}\left(x^{-1}\right)=\mu^{-}(x)$.

Definition 2.3. A bipolar L-fuzzy subset $\mu$ of $G$ is said to be bipolar L-fuzzy sub $\ell$ group of $\mathrm{G}$ if for any $\mathrm{x}, \mathrm{y} \in \mathrm{G}$.
i) $\quad \mu^{+}(\mathrm{xy}) \geq \mu^{+}(\mathrm{x}) \wedge \mu^{+}(\mathrm{y})$
ii) $\quad \mu^{-}(\mathrm{xy}) \leq \mu^{-}(\mathrm{x}) \vee \mu^{-}(\mathrm{y})$
iii) $\mu^{+}\left(\mathrm{x}^{-1}\right)=\mu^{+}(\mathrm{x})$
iv) $\mu^{-}\left(x^{-1}\right)=\mu^{-}(x)$
v) $\quad \mu^{+}(\mathrm{x} \vee \mathrm{y}) \geq \mu^{+}(\mathrm{x}) \wedge \mu^{+}(\mathrm{y})$
vi) $\quad \mu^{-}(\mathrm{x} \vee \mathrm{y}) \leq \mu^{-}(\mathrm{x}) \vee \mu^{-}(\mathrm{y})$
vii) $\quad \mu^{+}(\mathrm{x} \wedge \mathrm{y}) \geq \mu^{+}(\mathrm{x}) \wedge \mu^{+}(\mathrm{y})$
viii) $\quad \mu^{-}(\mathrm{x} \wedge \mathrm{y}) \leq \mu^{-}(\mathrm{x}) \vee \mu^{-}(\mathrm{y})$

Definition 2.4. A bipolar anti L-fuzzy subset $\mu$ of $G$ is said to be bipolar anti L-fuzzy sub $\ell$ group of $\mathrm{G}$ if for any $\mathrm{x}, \mathrm{y} \in \mathrm{G}$
i) $\quad \mu^{+}(\mathrm{xy}) \leq \mu^{+}(\mathrm{x}) \vee \mu^{+}(\mathrm{y})$
ii) $\quad \mu^{-}(\mathrm{xy}) \geq \mu^{-}(\mathrm{x}) \wedge \mu^{-}(\mathrm{y})$ 
Bipolar Anti L-Fuzzy Sub $\ell$-HX Group and its Lower Level Sub $\ell$-HX Groups

$\begin{array}{ll}\text { iii) } & \mu^{+}\left(x^{-1}\right)=\mu^{+}(x) \\ \text { iv) } & \mu^{-}\left(x^{-1}\right)=\mu^{-}(x) \\ \text { v) } & \mu^{+}(x \vee y) \leq \mu^{+}(x) \vee \mu^{+}(y) \\ \text { vi) } & \mu^{-}(x \vee y) \geq \mu^{-}(x) \wedge \mu^{-}(y) \\ \text { vii }) & \mu^{+}(x \wedge y) \leq \mu^{+}(x) \vee \mu^{+}(y) \\ \text { viii }) & \mu^{-}(x \wedge y) \geq \mu^{-}(x) \wedge \mu^{-}(y)\end{array}$

Definition 2.5. Let $\mu$ be a bipolar L - fuzzy subset defined on G. Let $\vartheta \subset 2^{\mathrm{G}}-\{\phi\}$ be a $\ell$ HX group on G. A bipolar L - fuzzy set $\lambda^{\mu}$ defined on $\vartheta$ is said to be a bipolar L - fuzzy sub $\ell-\mathrm{HX}$ group on $\vartheta$ if for all $\mathrm{A}, \mathrm{B} \in \vartheta$.

i) $\left(\lambda^{\mu}\right)^{+}(\mathrm{AB}) \geq\left(\lambda^{\mu}\right)^{+}(\mathrm{A}) \wedge\left(\lambda^{\mu}\right)^{+}(\mathrm{B})$

ii) $\left(\lambda^{\mu}\right)^{-}(\mathrm{AB}) \leq\left(\lambda^{\mu}\right)^{-}(\mathrm{A}) \vee\left(\lambda^{\mu}\right)^{-}(\mathrm{B})$

iii) $\left(\lambda^{\mu}\right)^{+}(\mathrm{A})=\left(\lambda^{\mu}\right)^{+}\left(\mathrm{A}^{-1}\right)$

iv) $\left(\lambda^{\mu}\right)^{-}(\mathrm{A})=\left(\lambda^{\mu}\right)^{-}\left(\mathrm{A}^{-1}\right)$

v) $\left(\lambda^{\mu}\right)^{+}(\mathrm{A} \vee \mathrm{B}) \geq\left(\lambda^{\mu}\right)^{+}(\mathrm{A}) \wedge\left(\lambda^{\mu}\right)^{+}(\mathrm{B})$

vi) $\left(\lambda^{\mu}\right)^{-}(\mathrm{A} \vee \mathrm{B}) \leq\left(\lambda^{\mu}\right)^{-}(\mathrm{A}) \vee\left(\lambda^{\mu}\right)^{-}(\mathrm{B})$

vii) $\left(\lambda^{\mu}\right)^{+}(\mathrm{A} \wedge \mathrm{B}) \geq\left(\lambda^{\mu}\right)^{+}(\mathrm{A}) \wedge\left(\lambda^{\mu}\right)^{+}(\mathrm{B})$

viii) $\left(\lambda^{\mu}\right)^{-}(\mathrm{A} \wedge \mathrm{B}) \leq\left(\lambda^{\mu}\right)^{-}(\mathrm{A}) \vee\left(\lambda^{\mu}\right)^{-}(\mathrm{B})$

where $\left(\lambda^{\mu}\right)^{+}(\mathrm{A})=\max \left\{\mu^{+}(\mathrm{x}) /\right.$ for all $\left.\mathrm{x} \in \mathrm{A} \subseteq \mathrm{G}\right\}$

and

$\left(\lambda^{\mu}\right)^{-}(\mathrm{A})=\min \left\{\mu^{-}(\mathrm{x}) /\right.$ for all $\left.\mathrm{x} \in \mathrm{A} \subseteq \mathrm{G}\right\}$

Example 2.1. Let $G=\left\{Z_{10}-\{0\}, \bullet_{10}\right\}$ be a group and define a bipolar L- fuzzy set $\mu$ on $G$ as $\mu^{+}(1)=0.7, \mu^{+}(3)=0.4, \mu^{+}(7)=0.4, \mu^{+}(9)=0.3$ and $\mu^{-}(1)=-0.8, \mu^{-}(3)=-0.3, \mu^{-}(7)=-0.3, \mu^{-}$ $(9)=-0.2$.

By routine computations, it is easy to see that $\mu$ is a bipolar L-fuzzy sub group of G.

Let $\vartheta=\{\{3,7\},\{1,9\}\}$ be a $\ell-$ HX group of $\mathrm{G}$.

Let us consider $\mathrm{A}=\{3,7\}, \mathrm{B}=\{1,9\}$.

\begin{tabular}{|c|c|c|}
\hline$\cdot 10$ & $\mathrm{~A}$ & $\mathrm{~B}$ \\
\hline $\mathrm{A}$ & $\mathrm{B}$ & $\mathrm{A}$ \\
\hline $\mathrm{B}$ & $\mathrm{A}$ & $\mathrm{B}$ \\
\hline
\end{tabular}

\begin{tabular}{|c|c|c|}
\hline$\wedge$ & $\mathrm{A}$ & $\mathrm{B}$ \\
\hline $\mathrm{A}$ & $\mathrm{A}$ & $\mathrm{B}$ \\
\hline $\mathrm{B}$ & $\mathrm{B}$ & $\mathrm{B}$ \\
\hline
\end{tabular}

\begin{tabular}{|c|c|c|}
\hline $\mathrm{V}$ & $\mathrm{A}$ & $\mathrm{B}$ \\
\hline $\mathrm{A}$ & $\mathrm{A}$ & $\mathrm{A}$ \\
\hline $\mathrm{B}$ & $\mathrm{A}$ & $\mathrm{B}$ \\
\hline
\end{tabular}

Define $\left(\lambda^{\mu}\right)^{+}(\mathrm{A})=\max \left\{\mu^{+}(\mathrm{x}) /\right.$ for all $\left.\mathrm{x} \in \mathrm{A} \subseteq \mathrm{G}\right\}$

And

$$
\left(\lambda^{\mu}\right)^{-}(\mathrm{A})=\min \left\{\mu^{-}(\mathrm{x}) / \text { for all } \mathrm{x} \in \mathrm{A} \subseteq \mathrm{G}\right\}
$$

Now $\left(\lambda^{\mu}\right)^{+}(A)=\left(\lambda^{\mu}\right)^{+}(\{3,7\})=\max \left\{\mu^{+}(3), \mu^{+}(7)\right\}=\max \{0.4,0.4\}=0.4$

$$
\begin{aligned}
& \left(\lambda^{\mu}\right)^{+}(\mathrm{B})=\left(\lambda^{\mu}\right)^{+}(\{1,9\})=\max \left\{\mu^{+}(1), \mu^{+}(9)\right\}=\max \{0.7,0.3\}=0.7 \\
& \left(\lambda^{\mu}\right)^{+}(\mathrm{AB})=\left(\lambda^{\mu}\right)^{+}(\mathrm{A})=0.4
\end{aligned}
$$


R. Muthuraj and G. Santha Meena

$$
\begin{aligned}
& \left(\lambda^{\mu}\right)^{+}(\mathrm{A} \wedge \mathrm{B})=\left(\lambda^{\mu}\right)^{+}(\mathrm{B})=0.7 \\
& \left(\lambda^{\mu}\right)^{+}(\mathrm{A} \vee \mathrm{B})=\left(\lambda^{\mu}\right)^{+}(\mathrm{A})=0.4 \\
& \left(\lambda^{\mu}\right)^{-}(\mathrm{A})=\left(\lambda^{\mu}\right)^{-}(\{3,7\})=\min \left\{\mu^{-}(3), \mu^{-}(7)\right\}=\min \{-0.3,-0.3\}=-0.3 \\
& \left(\lambda^{\mu}\right)^{-}(\mathrm{B})=\left(\lambda^{\mu}\right)^{-}(\{1,9\})=\min \left\{\mu^{-}(1), \mu^{-}(9)\right\}=\min \{-0.8,-0.2\}=-0.8 \\
& \left(\lambda^{\mu}\right)^{-}(\mathrm{AB})=\left(\lambda^{\mu}\right)^{-}(\mathrm{A})=-0.3 \\
& \left(\lambda^{\mu}\right)^{-}(\mathrm{A} \wedge \mathrm{B})=\left(\lambda^{\mu}\right)^{-}(\mathrm{B})=-0.8 \\
& \left(\lambda^{\mu}\right)^{-}(\mathrm{A} \vee \mathrm{B})=\left(\lambda^{\mu}\right)^{-}(\mathrm{A})=-0.3
\end{aligned}
$$

By routine computations, it is easy to see that $\lambda^{\mu}$ is a bipolar L-fuzzy sub $\ell-$ HX group of $\vartheta$.

Definition 2.6. Let $\mu$ be a bipolar L - fuzzy subset defined on G. Let $\vartheta \subset 2^{\mathrm{G}}-\{\phi\}$ be a $\ell$ HX group on G. A bipolar L - fuzzy set $\lambda^{\mu}$ defined on $\vartheta$ is said to be a bipolar anti L fuzzy sub $\ell-H X$ group on $\vartheta$ if for all $A, B \in \vartheta$.

$\begin{array}{ll}\text { i) } & \left(\lambda^{\mu}\right)^{+}(\mathrm{AB}) \leq\left(\lambda^{\mu}\right)^{+}(\mathrm{A}) \vee\left(\lambda^{\mu}\right)^{+}(\mathrm{B}) \\ \text { ii) } & \left(\lambda^{\mu}\right)^{-}(\mathrm{AB}) \geq\left(\lambda^{\mu}\right)^{-}(\mathrm{A}) \wedge\left(\lambda^{\mu}\right)^{-}(\mathrm{B}) \\ \text { iii) } & \left(\lambda^{\mu}\right)^{+}(\mathrm{A})=\left(\lambda^{\mu}\right)^{+}\left(\mathrm{A}^{-1}\right) \\ \text { iv) } & \left(\lambda^{\mu}\right)^{-}(\mathrm{A})=\left(\lambda^{\mu}\right)^{-}\left(\mathrm{A}^{-1}\right) \\ \text { v) } & \left(\lambda^{\mu}\right)^{+}(\mathrm{A} \vee \mathrm{B}) \leq\left(\lambda^{\mu}\right)^{+}(\mathrm{A}) \vee\left(\lambda^{\mu}\right)^{+}(\mathrm{B}) \\ \text { vi) } & \left(\lambda^{\mu}\right)^{-}(\mathrm{A} \vee \mathrm{B}) \geq\left(\lambda^{\mu}\right)^{-}(\mathrm{A}) \wedge\left(\lambda^{\mu}\right)^{-}(\mathrm{B}) \\ \text { vii) } & \left(\lambda^{\mu}\right)^{+}(\mathrm{A} \wedge \mathrm{B}) \leq\left(\lambda^{\mu}\right)^{+}(\mathrm{A}) \vee\left(\lambda^{\mu}\right)^{+}(\mathrm{B}) \\ \text { viii) } & \left(\lambda^{\mu}\right)^{-}(\mathrm{A} \wedge \mathrm{B}) \geq\left(\lambda^{\mu}\right)^{-}(\mathrm{A}) \wedge\left(\lambda^{\mu}\right)^{-}(\mathrm{B})\end{array}$

where $\left(\lambda^{\mu}\right)^{+}(\mathrm{A})=\min \left\{\mu^{+}(\mathrm{x}) /\right.$ for all $\left.\mathrm{x} \in \mathrm{A} \subseteq \mathrm{G}\right\}$ and

$$
\left(\lambda^{\mu}\right)^{-}(A)=\max \left\{\mu^{-}(\mathrm{x}) / \text { for all } \mathrm{x} \in \mathrm{A} \subseteq \mathrm{G}\right\}
$$

Example 2.2. Let $G=\left\{Z_{10^{-}}\{0\}, \cdot{ }_{10}\right\}$ be a group and define a bipolar L- fuzzy set $\mu$ on $G$ as $\mu^{+}(1)=0.3, \mu^{+}(3)=0.7, \mu^{+}(7)=0.7, \mu^{+}(9)=0.8$ and $\mu^{-}(1)=-0.4, \mu^{-}(3)=-0.6, \mu^{-}(7)=-0.6$, $\mu^{\prime}(9)=-0.7$.

By routine computations, it is easy to see that $\mu$ is a bipolar anti L-fuzzy sub group of G. Let $\vartheta=\{\{3,7\},\{1,9\}\}$ be a $\ell-\mathrm{HX}$ group of G.

Let us consider $A=\{3,7\}, B=\{1,9\}$.

\begin{tabular}{|c|c|c|}
\hline$\cdot 10$ & A & B \\
\hline A & B & A \\
\hline B & A & B \\
\hline
\end{tabular}

\begin{tabular}{|c|c|c|}
\hline$\wedge$ & $\mathrm{A}$ & $\mathrm{B}$ \\
\hline $\mathrm{A}$ & $\mathrm{A}$ & $\mathrm{B}$ \\
\hline $\mathrm{B}$ & $\mathrm{B}$ & $\mathrm{B}$ \\
\hline
\end{tabular}

\begin{tabular}{|c|c|c|}
\hline $\mathrm{V}$ & $\mathrm{A}$ & $\mathrm{B}$ \\
\hline $\mathrm{A}$ & $\mathrm{A}$ & $\mathrm{A}$ \\
\hline $\mathrm{B}$ & $\mathrm{A}$ & $\mathrm{B}$ \\
\hline
\end{tabular}


Bipolar Anti L-Fuzzy Sub $\ell$-HX Group and its Lower Level Sub $\ell$-HX Groups $\operatorname{Define}\left(\lambda^{\mu}\right)^{+}(\mathrm{A})=\min \left\{\mu^{+}(\mathrm{x}) /\right.$ for all $\left.\mathrm{x} \in \mathrm{A} \subseteq \mathrm{G}\right\}$

and

$\left(\lambda^{\mu}\right)^{-}(\mathrm{A})=\max \left\{\mu^{-}(\mathrm{x}) /\right.$ for all $\left.\mathrm{x} \in \mathrm{A} \subseteq \mathrm{G}\right\}$

Now $\left(\lambda^{\mu}\right)^{+}(A)=\left(\lambda^{\mu}\right)^{+}(\{3,7\})=\min \left\{\mu^{+}(3), \mu^{+}(7)\right\}=\min \{0.7,0.7\}=0.7$

$\left(\lambda^{\mu}\right)^{+}(B)=\left(\lambda^{\mu}\right)^{+}(\{1,9\})=\min \left\{\mu^{+}(1), \mu^{+}(9)\right\}=\min \{0.3,0.8\}=0.3$

$\left(\lambda^{\mu}\right)^{+}(\mathrm{AB})=\left(\lambda^{\mu}\right)^{+}(\mathrm{A})=0.7$

$\left(\lambda^{\mu}\right)^{+}(\mathrm{A} \wedge \mathrm{B})=\left(\lambda^{\mu}\right)^{+}(\mathrm{B})=0.3$

$\left(\lambda^{\mu}\right)^{+}(\mathrm{A} \vee \mathrm{B})=\left(\lambda^{\mu}\right)^{+}(\mathrm{A})=0.7$

$\left(\lambda^{\mu}\right)^{-}(\mathrm{A})=\left(\lambda^{\mu}\right)^{-}(\{3,7\})=\max \left\{\mu^{-}(3), \mu^{-}(7)\right\}=\max \{-0.6,-0.6\}=-0.6$

$\left(\lambda^{\mu}\right)^{-}(B)=\left(\lambda^{\mu}\right)^{-}(\{1,9\})=\max \left\{\mu^{-}(1), \mu^{-}(9)\right\}=\max \{-0.4,-0.7\}=-0.4$

$\left(\lambda^{\mu}\right)^{-}(\mathrm{AB})=\left(\lambda^{\mu}\right)^{-}(\mathrm{A})=-0.6$

$\left(\lambda^{\mu}\right)^{-}(\mathrm{A} \wedge \mathrm{B})=\left(\lambda^{\mu}\right)^{-}(\mathrm{B})=-0.4$

$\left(\lambda^{\mu}\right)^{-}(\mathrm{A} \vee \mathrm{B})=\left(\lambda^{\mu}\right)^{-}(\mathrm{A})=-0.6$

By routine computations, it is easy to see that $\lambda^{\mu}$ is a bipolar anti L-fuzzy sub $\ell-\mathrm{HX}$ group of $\vartheta$.

\section{Properties of bipolar anti L-fuzzy sub $\ell$ - HX group}

In this section, we discuss some of the properties ofbipolar anti L-fuzzy sub $\ell-\mathrm{HX}$ group.

Theorem 3.1. Let $\mathrm{G}$ be a group. If $\mu$ is a bipolar anti L-fuzzy sub $\ell$ group of $G$ then the bipolar L-fuzzy set $\lambda^{\mu}$ is a bipolar anti L-fuzzy sub $\ell$ - HX group of $\vartheta$.

Proof: Let $\mu$ be a bipolar anti L-fuzzy sub $\ell$ group of $\mathrm{G}$ and $\lambda^{\mu}$ be a bipolar L-fuzzy subset on $\mathrm{G}$ for any $\mathrm{A}, \mathrm{B} \in \vartheta \subset \mathrm{G}$

i) $\left(\lambda^{\mu}\right)^{+}(\mathrm{A}) \vee\left(\lambda^{\mu}\right)^{+}(\mathrm{B})=\min \left\{\mu^{+}(\mathrm{x}) /\right.$ for all $\left.\mathrm{x} \in \mathrm{A} \subset \mathrm{G}\right\} \vee \min \left\{\mu^{+}(\mathrm{y}) /\right.$ for all $\left.\mathrm{y} \in \mathrm{B} \subset \mathrm{G}\right\}$

$=\mu^{+}\left(\mathrm{x}_{0}\right) \vee \mu^{+}\left(\mathrm{y}_{0}\right)$, some $\mathrm{x}_{0} \in \mathrm{A}, \mathrm{y}_{0} \in \mathrm{B}$ and $\mathrm{A}, \mathrm{B} \subset \mathrm{G}$

$\geq \mu^{+}\left(\mathrm{x}_{0} \mathrm{y}_{0}\right), \mu$ is a bipolar anti L-fuzzy sub $\ell$ group on $\mathrm{G}$

$=\min \left\{\mu^{+}(\mathrm{xy}) /\right.$ for all $\mathrm{x} \in \mathrm{A}, \mathrm{y} \in \mathrm{B}$ and $\left.\mathrm{A}, \mathrm{B} \subset \mathrm{G}\right\}$

$=\left(\lambda^{\mu}\right)^{+}(\mathrm{AB})$.

So, $\left(\lambda^{\mu}\right)^{+}(\mathrm{AB}) \leq\left(\lambda^{\mu}\right)^{+}(\mathrm{A}) \vee\left(\lambda^{\mu}\right)^{+}(\mathrm{B})$

ii) $\left(\lambda^{\mu}\right)^{-}(\mathrm{A}) \wedge\left(\lambda^{\mu}\right)^{-}(\mathrm{B})=\max \left\{\mu^{-}(\mathrm{x}) /\right.$ for all $\left.\mathrm{x} \in \mathrm{A} \subset \mathrm{G}\right\} \wedge \max \left\{\mu^{-}(\mathrm{y}) /\right.$ for all $\left.\mathrm{y} \in \mathrm{B} \subset \mathrm{G}\right\}$

$$
=\mu^{-}\left(\mathrm{x}_{0}\right) \wedge \mu^{-}\left(\mathrm{y}_{0}\right) \text {, some } \mathrm{x}_{0} \in \mathrm{A}, \mathrm{y}_{0} \in \mathrm{B} \text { and } \mathrm{A}, \mathrm{B} \subset \mathrm{G}
$$


R. Muthuraj and G. Santha Meena

$\leq \mu^{-}\left(\mathrm{x}_{0} \mathrm{y}_{0}\right), \mu$ is a bipolar anti L-fuzzy sub $\ell$ group on $\mathrm{G}$

$=\max \left\{\mu^{+}(\mathrm{xy}) /\right.$ for all $\mathrm{x} \in \mathrm{A}, \mathrm{y} \in \mathrm{B}$ and $\left.\mathrm{A}, \mathrm{B} \subset \mathrm{G}\right\}$

$=\left(\lambda^{\mu}\right)^{-}(\mathrm{AB})$

So, $\left(\lambda^{\mu}\right)^{-}(\mathrm{AB}) \quad \geq\left(\lambda^{\mu}\right)^{+}(\mathrm{A}) \wedge\left(\lambda^{\mu}\right)^{+}(\mathrm{B})$

iii) $\quad\left(\lambda^{\mu}\right)^{+}(\mathrm{A})=\min \left\{\mu^{+}(\mathrm{x}) /\right.$ for all $\left.\mathrm{x} \in \mathrm{A} \subset \mathrm{G}\right\}$

$=\min \left\{\mu^{+}\left(\mathrm{x}^{-1}\right) /\right.$ for all $\left.\mathrm{x}^{-1} \in \mathrm{A} \subset \mathrm{G}\right\}$

$=\min \left\{\mu^{+}\left(\mathrm{x}^{-1}\right) /\right.$ for all $\left.\mathrm{x}^{-1} \in \mathrm{A}^{-1} \subset \mathrm{G}\right\}$

So, $\left(\lambda^{\mu}\right)^{+}(\mathrm{A})=\left(\lambda^{\mu}\right)^{+}\left(\mathrm{A}^{-1}\right)$

iv) $\quad\left(\lambda^{\mu}\right)^{-}(\mathrm{A})=\max \left\{\mu^{-}(\mathrm{x}) /\right.$ for all $\left.\mathrm{x} \in \mathrm{A} \subset \mathrm{G}\right\}$

$=\max \left\{\mu^{-}\left(\mathrm{x}^{-1}\right) /\right.$ for all $\left.\mathrm{x}^{-1} \in \mathrm{A} \subset \mathrm{G}\right\}$

$=\max \left\{\mu^{-}\left(\mathrm{x}^{-1}\right) /\right.$ for all $\left.\mathrm{x}^{-1} \in \mathrm{A}^{-1} \subset \mathrm{G}\right\}$

So, $\left(\lambda^{\mu}\right)^{-}(\mathrm{A})=\left(\lambda^{\mu}\right)^{-}\left(\mathrm{A}^{-1}\right)$

v) $\left(\lambda^{\mu}\right)^{+}(A) \vee\left(\lambda^{\mu}\right)^{+}(B)=\min \left\{\mu^{+}(x) /\right.$ for all $\left.x \in A \subset G\right\} \vee \min \left\{\mu^{+}(y) /\right.$ for all $\left.y \in B \subset G\right\}$

$=\mu^{+}\left(x_{0}\right) \vee \mu^{+}\left(y_{0}\right)$, some $x_{0} \in A, y_{0} \in B$ and $A, B \subset G$

$\geq \mu^{+}\left(x_{0} \vee y_{0}\right), \mu$ is a bipolar anti L-fuzzy sub $\ell$ group on $G$

$=\min \left\{\mu^{+}(x \vee y) /\right.$ for all $x \in A, y \in B$ and $\left.A, B \subset G\right\}$

$=\left(\lambda^{\mu}\right)^{+}(\mathrm{A} \vee \mathrm{B})$

So, $\left(\lambda^{\mu}\right)^{+}(\mathrm{A} \vee \mathrm{B}) \leq\left(\lambda^{\mu}\right)^{+}(\mathrm{A}) \vee\left(\lambda^{\mu}\right)^{+}(\mathrm{B})$

vi) $\left(\lambda^{\mu}\right)^{-}(\mathrm{A}) \wedge\left(\lambda^{\mu}\right)^{-}(\mathrm{B})=\max \left\{\mu^{-}(\mathrm{x}) /\right.$ for all $\left.\mathrm{x} \in \mathrm{A} \subset \mathrm{G}\right\} \wedge \max \left\{\mu^{-}(\mathrm{y}) /\right.$ for all $\left.\mathrm{y} \in \mathrm{B} \subset \mathrm{G}\right\}$

$=\mu^{-}\left(\mathrm{x}_{0}\right) \wedge \mu^{-}\left(\mathrm{y}_{0}\right)$, some $\mathrm{x}_{0} \in \mathrm{A}, \mathrm{y}_{0} \in \mathrm{B}$ and $\mathrm{A}, \mathrm{B} \subset \mathrm{G}$

$\leq \mu^{-}\left(\mathrm{x}_{0} \vee \mathrm{y}_{0}\right), \mu$ is a bipolar anti L-fuzzy sub $\ell$ group on $\mathrm{G}$

$=\max \left\{\mu^{-}(\mathrm{x} \vee \mathrm{y}) /\right.$ for all $\mathrm{x} \in \mathrm{A}, \mathrm{y} \in \mathrm{B}$ and $\left.\mathrm{A}, \mathrm{B} \subset \mathrm{G}\right\}$

$=\left(\lambda^{\mu}\right)^{-}(\mathrm{A} \vee \mathrm{B})$

So, $\left(\lambda^{\mu}\right)^{-}(A \vee B) \geq\left(\lambda^{\mu}\right)^{-}(A) \wedge\left(\lambda^{\mu}\right)^{-}(B)$

$\operatorname{vii})\left(\lambda^{\mu}\right)^{+}(\mathrm{A}) \vee\left(\lambda^{\mu}\right)^{+}(\mathrm{B})=\min \left\{\mu^{+}(\mathrm{x}) /\right.$ for all $\left.\mathrm{x} \in \mathrm{A} \subset \mathrm{G}\right\} \vee \min \left\{\mu^{+}(\mathrm{y}) /\right.$ for all $\left.\mathrm{y} \in \mathrm{B} \subset \mathrm{G}\right\}$

$=\mu^{+}\left(\mathrm{x}_{0}\right) \vee \mu^{+}\left(\mathrm{y}_{0}\right)$, some $\mathrm{x}_{0} \in \mathrm{A}, \mathrm{y}_{0} \in \mathrm{B}$ and $\mathrm{A}, \mathrm{B} \subset \mathrm{G}$

$\geq \mu^{+}\left(x_{0} \wedge y_{0}\right), \mu$ is a bipolar anti L-fuzzy sub $\ell$ group on $G$

$=\min \left\{\mu^{+}(\mathrm{x} \wedge \mathrm{y}) /\right.$ for all $\mathrm{x} \in \mathrm{A}, \mathrm{y} \in \mathrm{B}$ and $\left.\mathrm{A}, \mathrm{B} \subset \mathrm{G}\right\}$

$=\left(\lambda^{\mu}\right)^{+}(\mathrm{A} \wedge \mathrm{B})$ 
Bipolar Anti L-Fuzzy Sub $\ell$-HX Group and its Lower Level Sub $\ell$-HX Groups So, $\left(\lambda^{\mu}\right)^{+}(\mathrm{A} \wedge \mathrm{B}) \leq\left(\lambda^{\mu}\right)^{+}(\mathrm{A}) \vee\left(\lambda^{\mu}\right)^{+}(\mathrm{B})$ $\operatorname{viii)}\left(\lambda^{\mu}\right)^{-}(\mathrm{A}) \wedge\left(\lambda^{\mu}\right)^{-}(\mathrm{B})=\max \left\{\mu^{-}(\mathrm{x}) /\right.$ for all $\left.\mathrm{x} \in \mathrm{A} \subset \mathrm{G}\right\} \wedge \max \left\{\mu^{-}(\mathrm{y}) /\right.$ for all $\left.\mathrm{y} \in \mathrm{B} \subset \mathrm{G}\right\}$ $=\mu^{-}\left(\mathrm{x}_{0}\right) \wedge \mu^{-}\left(\mathrm{y}_{0}\right)$, some $\mathrm{x}_{0} \in \mathrm{A}, \mathrm{y}_{0} \in \mathrm{B}$ and $\mathrm{A}, \mathrm{B} \subset \mathrm{G}$ $\leq \mu^{-}\left(\mathrm{x}_{0} \wedge \mathrm{y}_{0}\right), \mu$ is a bipolar anti L-fuzzy sub $\ell$ group on $\mathrm{G}$ $=\max \left\{\mu^{-}(\mathrm{x} \wedge \mathrm{y}) /\right.$ for all $\mathrm{x} \in \mathrm{A}, \mathrm{y} \in \mathrm{B}$ and $\left.\mathrm{A}, \mathrm{B} \subset \mathrm{G}\right\}$ $=\left(\lambda^{\mu}\right)^{-}(\mathrm{A} \wedge \mathrm{B})$

So, $\left(\lambda^{\mu}\right)^{-}(\mathrm{A} \wedge \mathrm{B}) \quad \geq\left(\lambda^{\mu}\right)^{-}(\mathrm{A}) \wedge\left(\lambda^{\mu}\right)^{-}(\mathrm{B})$

Hence $\lambda^{\mu}$ is a bipolar anti L-fuzzy sub $\ell$ - HX group of $\vartheta$.

Theorem 3.2. Let $\lambda^{\mu}=\left(\left(\lambda^{\mu}\right)^{+},\left(\lambda^{\mu}\right)^{-}\right)$be a bipolar anti L-fuzzy sub $\ell$ - HX group of a $\ell$ - HX group $\vartheta$ then

i) $\quad\left(\lambda^{\mu}\right)^{+}(\mathrm{A}) \geq\left(\lambda^{\mu}\right)^{+}(\mathrm{E})$ and $\left(\lambda^{\mu}\right)^{-}(\mathrm{A}) \leq\left(\lambda^{\mu}\right)^{-}(\mathrm{E})$ for all $\mathrm{A} \in \vartheta$ and $\mathrm{E}$ is the identity element of $\vartheta$.

ii) The subset $\mathrm{H}=\left\{\mathrm{A} \in \vartheta /\left(\lambda^{\mu}\right)^{+}(\mathrm{A})=\left(\lambda^{\mu}\right)^{+}(\mathrm{E})\right.$ and $\left.\left(\lambda^{\mu}\right)^{-}(\mathrm{A})=\left(\lambda^{\mu}\right)^{-}(\mathrm{E})\right\}$ is a sub $\ell$ HX group of $\vartheta$.

Proof i) Let $A \in \vartheta$

$$
\begin{aligned}
\left(\lambda^{\mu}\right)^{+}(\mathrm{E}) \quad & =\left(\lambda^{\mu}\right)^{+}\left(\mathrm{A} \mathrm{A}^{-1}\right) \\
& \leq\left(\lambda^{\mu}\right)^{+}(\mathrm{A}) \vee\left(\lambda^{\mu}\right)^{+}\left(\mathrm{A}^{-1}\right) \\
= & \left(\lambda^{\mu}\right)^{+}(\mathrm{A}) \vee\left(\lambda^{\mu}\right)^{+}(\mathrm{A}) \\
& =\left(\lambda^{\mu}\right)^{+}(\mathrm{A})
\end{aligned}
$$

Therefore, $\left(\lambda^{\mu}\right)^{+}(\mathrm{A}) \geq\left(\lambda^{\mu}\right)^{+}(\mathrm{E})$, for all $\mathrm{A} \in \vartheta$

Similarly, for all $\mathrm{A} \in \vartheta$

$$
\begin{aligned}
\left(\lambda^{\mu}\right)^{-}(\mathrm{E}) & =\left(\lambda^{\mu}\right)^{-}\left(\mathrm{A} \mathrm{A}{ }^{-1}\right) \\
& \geq\left(\lambda^{\mu}\right)^{-}(\mathrm{A}) \wedge\left(\lambda^{\mu}\right)^{-}\left(\mathrm{A}^{-1}\right) \\
& =\left(\lambda^{\mu}\right)^{-}(\mathrm{A}) \wedge\left(\lambda^{\mu}\right)^{-}(\mathrm{A}) \\
& =\left(\lambda^{\mu}\right)^{-}(\mathrm{A})
\end{aligned}
$$

Therefore, $\left(\lambda^{\mu}\right)^{-}(A) \leq\left(\lambda^{\mu}\right)^{-}(E)$, for all $A \in \vartheta$

ii)Let $H=\left\{A \in \vartheta /\left(\lambda^{\mu}\right)^{+}(A)=\left(\lambda^{\mu}\right)^{+}(E)\right.$ and $\left.\left(\lambda^{\mu}\right)^{-}(A)=\left(\lambda^{\mu}\right)^{-}(E)\right\}$ clearly, $H$ is non-empty as E $\in$ H Let $A, B \in H$, then $\left(\lambda^{\mu}\right)^{+}(A)=\left(\lambda^{\mu}\right)^{+}(B)=\left(\lambda^{\mu}\right)^{+}(E)$ and $\left(\lambda^{\mu}\right)^{-}(A)=\left(\lambda^{\mu}\right)^{-}(B)=\left(\lambda^{\mu}\right)^{-}(E)$ Now, $\left(\lambda^{\mu}\right)^{+}\left(\mathrm{AB}^{-1}\right) \leq\left(\lambda^{\mu}\right)^{+}(\mathrm{A}) \vee\left(\lambda^{\mu}\right)^{+}\left(\mathrm{B}^{-1}\right)$ 
R. Muthuraj and G. Santha Meena

$=\left(\lambda^{\mu}\right)^{+}(\mathrm{A}) \vee\left(\lambda^{\mu}\right)^{+}(\mathrm{B})$

$=\left(\lambda^{\mu}\right)^{+}(\mathrm{E}) \vee\left(\lambda^{\mu}\right)^{+}(\mathrm{E})$

$=\left(\lambda^{\mu}\right)^{+}(\mathrm{E})$

$$
\left(\lambda^{\mu}\right)^{+}\left(\mathrm{AB}^{-1}\right) \leq\left(\lambda^{\mu}\right)^{+}(\mathrm{E})
$$

That is, $\left(\lambda^{\mu}\right)^{+}\left(\mathrm{AB}^{-1}\right) \leq\left(\lambda^{\mu}\right)^{+}(\mathrm{E})$ and obviously $\left(\lambda^{\mu}\right)^{+}\left(\mathrm{AB}^{-1}\right) \geq\left(\lambda^{\mu}\right)^{+}(\mathrm{E})$

Hence, $\quad\left(\lambda^{\mu}\right)^{+}\left(\mathrm{AB}^{-1}\right)=\left(\lambda^{\mu}\right)^{+}(\mathrm{E})$ then $\mathrm{AB}^{-1} \in \mathrm{H}$

Now, $\quad\left(\lambda^{\mu}\right)^{-}\left(\mathrm{AB}^{-1}\right) \geq\left(\lambda^{\mu}\right)^{-}(\mathrm{A}) \wedge\left(\lambda^{\mu}\right)^{-}\left(\mathrm{B}^{-1}\right)$

$=\left(\lambda^{\mu}\right)^{-}(\mathrm{A}) \wedge\left(\lambda^{\mu}\right)^{-}(\mathrm{B})$

$=\left(\lambda^{\mu}\right)^{-}(\mathrm{E}) \wedge\left(\lambda^{\mu}\right)^{-}(\mathrm{E})$

$=\left(\lambda^{\mu}\right)^{-}(\mathrm{E})$

$$
\left(\lambda^{\mu}\right)^{-}\left(\mathrm{AB}^{-1}\right) \geq\left(\lambda^{\mu}\right)^{-}(\mathrm{E})
$$

That is, $\quad\left(\lambda^{\mu}\right)^{-(}\left(\mathrm{AB}^{-1}\right) \geq\left(\lambda^{\mu}\right)^{-(}(\mathrm{E})$ and obviously $\left(\lambda^{\mu}\right)^{-}\left(\mathrm{AB}^{-1}\right) \leq\left(\lambda^{\mu}\right)^{-}(\mathrm{E})$

Hence, $\quad\left(\lambda^{\mu}\right)^{-}\left(\mathrm{AB}^{-1}\right)=\left(\lambda^{\mu}\right)^{-}(\mathrm{E})$

Hence, $\quad\left(\lambda^{\mu}\right)^{-}\left(\mathrm{AB}^{-1}\right)=\left(\lambda^{\mu}\right)^{-}(\mathrm{E})$ then $\mathrm{AB}^{-1} \in \mathrm{H}$

Now, $\quad\left(\lambda^{\mu}\right)^{+}(\mathrm{A} \vee \mathrm{B}) \leq\left(\lambda^{\mu}\right)^{+}(\mathrm{A}) \vee\left(\lambda^{\mu}\right)^{+}(\mathrm{B})$

$$
\begin{aligned}
& =\left(\lambda^{\mu}\right)^{+}(\mathrm{A}) \vee\left(\lambda^{\mu}\right)^{+}(\mathrm{B}) \\
= & \left(\lambda^{\mu}\right)^{+}(\mathrm{E}) \vee\left(\lambda^{\mu}\right)^{+}(\mathrm{E}) \\
= & \left(\lambda^{\mu}\right)^{+}(\mathrm{E})
\end{aligned}
$$

$\left(\lambda^{\mu}\right)^{+}(\mathrm{A} \vee \mathrm{B}) \leq\left(\lambda^{\mu}\right)^{+}(\mathrm{E})$

That is, $\left(\lambda^{\mu}\right)^{+}(\mathrm{A} \vee \mathrm{B}) \leq\left(\lambda^{\mu}\right)^{+}(\mathrm{E})$ and obviously $\left(\lambda^{\mu}\right)^{+}(\mathrm{A} \vee \mathrm{B}) \geq\left(\lambda^{\mu}\right)^{+}(\mathrm{E})$

Hence, $\quad\left(\lambda^{\mu}\right)^{+}(\mathrm{A} \vee \mathrm{B})=\left(\lambda^{\mu}\right)^{+}(\mathrm{E})$ then $\mathrm{A} \vee \mathrm{B} \in \mathrm{H}$

Now, $\quad\left(\lambda^{\mu}\right)^{-}(\mathrm{A} \vee \mathrm{B}) \geq\left(\lambda^{\mu}\right)^{-}(\mathrm{A}) \wedge\left(\lambda^{\mu}\right)^{-}(\mathrm{B})$

$$
\begin{aligned}
& =\left(\lambda^{\mu}\right)^{-}(\mathrm{A}) \wedge\left(\lambda^{\mu}\right)^{-(}(\mathrm{B}) \\
& =\left(\lambda^{\mu}\right)^{-}(\mathrm{E}) \wedge\left(\lambda^{\mu}\right)^{-}(\mathrm{E}) \\
& =\left(\lambda^{\mu}\right)^{-}(\mathrm{E})
\end{aligned}
$$

$\left(\lambda^{\mu}\right)^{-}(\mathrm{A} \vee \mathrm{B}) \geq\left(\lambda^{\mu}\right)^{-}(\mathrm{E})$

That is, $\quad\left(\lambda^{\mu}\right)^{-}(\mathrm{A} \vee \mathrm{B}) \geq\left(\lambda^{\mu}\right)^{-}(\mathrm{E})$ and obviously $\left(\lambda^{\mu}\right)^{-}(\mathrm{A} \vee \mathrm{B}) \leq\left(\lambda^{\mu}\right)^{-}(\mathrm{E})$

Hence, $\quad\left(\lambda^{\mu}\right)^{-}(\mathrm{A} \vee \mathrm{B})=\left(\lambda^{\mu}\right)^{-}(\mathrm{E})$ then $\mathrm{A} \vee \mathrm{B} \in \mathrm{H}$

Now, $\quad\left(\lambda^{\mu}\right)^{+}(\mathrm{A} \wedge \mathrm{B}) \leq\left(\lambda^{\mu}\right)^{+}(\mathrm{A}) \vee\left(\lambda^{\mu}\right)^{+}(\mathrm{B})$ 
Bipolar Anti L-Fuzzy Sub $\ell$-HX Group and its Lower Level Sub $\ell$-HX Groups

$$
\begin{aligned}
&=\left(\lambda^{\mu}\right)^{+}(\mathrm{A}) \vee\left(\lambda^{\mu}\right)^{+}(\mathrm{B}) \\
&=\left(\lambda^{\mu}\right)^{+}(\mathrm{E}) \vee\left(\lambda^{\mu}\right)^{+}(\mathrm{E}) \\
&=\left(\lambda^{\mu}\right)^{+}(\mathrm{E}) \\
&\left(\lambda^{\mu}\right)^{+}(\mathrm{A} \wedge \mathrm{B}) \leq\left(\lambda^{\mu}\right)^{+}(\mathrm{E})
\end{aligned}
$$

That is, $\quad\left(\lambda^{\mu}\right)^{+}(\mathrm{A} \wedge \mathrm{B}) \leq\left(\lambda^{\mu}\right)^{+}(\mathrm{E})$ and obviously $\left(\lambda^{\mu}\right)^{+}(\mathrm{A} \wedge \mathrm{B}) \geq\left(\lambda^{\mu}\right)^{+}(\mathrm{E})$

Hence, $\quad\left(\lambda^{\mu}\right)^{+}(\mathrm{A} \wedge \mathrm{B})=\left(\lambda^{\mu}\right)^{+}(\mathrm{E})$ then $\mathrm{A} \wedge \mathrm{B} \in \mathrm{H}$

Now, $\quad\left(\lambda^{\mu}\right)^{-}(\mathrm{A} \wedge \mathrm{B}) \geq\left(\lambda^{\mu}\right)^{-(}(\mathrm{A}) \wedge\left(\lambda^{\mu}\right)^{-}(\mathrm{B})$

$$
\begin{aligned}
& =\left(\lambda^{\mu}\right)^{-}(\mathrm{A}) \wedge\left(\lambda^{\mu}\right)^{-(}(\mathrm{B}) \\
& =\left(\lambda^{\mu}\right)^{-}(\mathrm{E}) \wedge\left(\lambda^{\mu}\right)^{-}(\mathrm{E}) \\
& =\left(\lambda^{\mu}\right)^{-}(\mathrm{E})
\end{aligned}
$$

$$
\left(\lambda^{\mu}\right)^{-}(\mathrm{A} \wedge \mathrm{B}) \geq\left(\lambda^{\mu}\right)^{-}(\mathrm{E})
$$

That is, $\quad\left(\lambda^{\mu}\right)^{-}(\mathrm{A} \wedge \mathrm{B}) \geq\left(\lambda^{\mu}\right)^{-}(\mathrm{E})$ and obviously $\left(\lambda^{\mu}\right)^{-}(\mathrm{A} \wedge \mathrm{B}) \leq\left(\lambda^{\mu}\right)^{-}(\mathrm{E})$

Hence, $\quad\left(\lambda^{\mu}\right)^{-}(\mathrm{A} \wedge \mathrm{B})=\left(\lambda^{\mu}\right)^{-}(\mathrm{E})$ then $\mathrm{A} \wedge \mathrm{B} \in \mathrm{H}$

Clearly, $\mathrm{H}$ is a sub $\ell-\mathrm{HX}$ group of $\vartheta$.

Theorem 3.3. Let $\lambda^{\mu}=\left(\left(\lambda^{\mu}\right)^{+},\left(\lambda^{\mu}\right)^{-}\right)$be a bipolar anti L-fuzzy sub $\ell$ - HX group of a $\ell$ - HX group $\vartheta$ with identity E. Then

i) $\quad\left(\lambda^{\mu}\right)^{+}\left(\mathrm{AB}^{-1}\right)=\left(\lambda^{\mu}\right)^{+}(\mathrm{E}) \Rightarrow\left(\lambda^{\mu}\right)^{+}(\mathrm{A})=\left(\lambda^{\mu}\right)^{+}(\mathrm{B})$, for all A,B in $\vartheta$

ii) $\quad\left(\lambda^{\mu}\right)^{-}\left(\mathrm{AB}^{-1}\right)=\left(\lambda^{\mu}\right)^{-}(\mathrm{E}) \Rightarrow\left(\lambda^{\mu}\right)^{-}(\mathrm{A})=\left(\lambda^{\mu}\right)^{-}(\mathrm{B})$, for all A,B in $\vartheta$

Proof: i) Let $\lambda^{\mu}=\left(\left(\lambda^{\mu}\right)^{+},\left(\lambda^{\mu}\right)^{-}\right)$be a bipolar anti L - fuzzy sub $\ell$ - HX group of a $\ell$ - HX group $\vartheta$ with identity $E$ and $\left(\lambda^{\mu}\right)^{+}\left(\mathrm{AB}^{-1}\right)=\left(\lambda^{\mu}\right)^{+}(\mathrm{E})$ Then, for all $\mathrm{A}, \mathrm{B}$ in $\vartheta$.

$$
\begin{aligned}
\left(\lambda^{\mu}\right)^{+}(\mathrm{A}) & =\left(\lambda^{\mu}\right)^{+}\left(\mathrm{A}\left(\mathrm{B}^{-1} \mathrm{~B}\right)\right) \\
& =\left(\lambda^{\mu}\right)^{+}\left(\left(\mathrm{A}\left(\mathrm{B}^{-1}\right) \mathrm{B}\right)\right. \\
& \leq\left(\lambda^{\mu}\right)^{+}\left(\mathrm{AB}{ }^{-1}\right) \vee\left(\lambda^{\mu}\right)^{+}(\mathrm{B}) \\
& =\left(\lambda^{\mu}\right)^{+}(\mathrm{E}) \vee\left(\lambda^{\mu}\right)^{+}(\mathrm{B}) \\
& =\left(\lambda^{\mu}\right)^{+}(\mathrm{B})
\end{aligned}
$$

That is, $\left(\lambda^{\mu}\right)^{+}(\mathrm{A}) \leq\left(\lambda^{\mu}\right)^{+}(\mathrm{B})$

That is, $\left(\lambda^{\mu}\right)^{+}\left(\mathrm{AB}^{-1}\right)=\left(\lambda^{\mu}\right)^{+}(\mathrm{E}) \Rightarrow\left(\lambda^{\mu}\right)^{+}(\mathrm{A}) \leq\left(\lambda^{\mu}\right)^{+}(\mathrm{B})$

Since, $\left(\lambda^{\mu}\right)^{+}\left(\mathrm{BA}^{-1}\right)=\left(\lambda^{\mu}\right)^{+}\left(\left(\mathrm{BA}^{-1}\right)^{-1}\right)$

$$
=\left(\lambda^{\mu}\right)^{+}\left(\mathrm{AB}^{-1}\right)
$$


R. Muthuraj and G. Santha Meena

$$
\begin{array}{r}
=\left(\lambda^{\mu}\right)^{+}(\mathrm{E}) \\
\left(\lambda^{\mu}\right)^{+}\left(\mathrm{BA}^{-1}\right)=\left(\lambda^{\mu}\right)^{+}(\mathrm{E})
\end{array}
$$

That is, $\quad\left(\lambda^{\mu}\right)^{+}\left(\mathrm{BA}^{-1}\right)=\left(\lambda^{\mu}\right)^{+}(\mathrm{E}) \Rightarrow\left(\lambda^{\mu}\right)^{+}(\mathrm{B}) \leq\left(\lambda^{\mu}\right)^{+}(\mathrm{A})$

Hence, $\quad\left(\lambda^{\mu}\right)^{+}(\mathrm{A})=\left(\lambda^{\mu}\right)^{+}(\mathrm{B})$

Therefore, $\left(\lambda^{\mu}\right)^{+}\left(\mathrm{AB}^{-1}\right)=\left(\lambda^{\mu}\right)^{+}(\mathrm{E}) \Rightarrow\left(\lambda^{\mu}\right)^{+}(\mathrm{A})=\left(\lambda^{\mu}\right)^{+}(\mathrm{B})$, for all A,B in $\vartheta$.

ii)Let $\lambda^{\mu}=\left(\left(\lambda^{\mu}\right)^{+},\left(\lambda^{\mu}\right)^{-}\right)$be a bipolar anti L-fuzzy sub $\ell$ - HX group of a $\ell$ - HX group $\vartheta$ withidentity $\mathrm{E}$ and $\left(\lambda^{\mu}\right)^{-}\left(\mathrm{AB}^{-1}\right)=\left(\lambda^{\mu}\right)^{-}(\mathrm{E})$ Then, for all $\mathrm{A}, \mathrm{B}$ in $\vartheta$

$$
\begin{aligned}
\left(\lambda^{\mu}\right)^{-(}(\mathrm{A}) & =\left(\lambda^{\mu}\right)^{-}\left(\mathrm{A}\left(\mathrm{B}^{-1} \mathrm{~B}\right)\right) \\
& =\left(\lambda^{\mu}\right)^{-}\left(\left(\mathrm{A}\left(\mathrm{B}^{-1}\right) \mathrm{B}\right)\right. \\
\geq & \left(\lambda^{\mu}\right)^{-}\left(\mathrm{AB}^{-1}\right) \wedge\left(\lambda^{\mu}\right)^{-(}(\mathrm{B}) \\
& =\left(\lambda^{\mu}\right)^{-}(\mathrm{E}) \wedge\left(\lambda^{\mu}\right)^{-}(\mathrm{B}) \\
& =\left(\lambda^{\mu}\right)^{-}(\mathrm{B})
\end{aligned}
$$

That is, $\quad\left(\lambda^{\mu}\right)^{-}(\mathrm{A}) \geq\left(\lambda^{\mu}\right)^{-}(\mathrm{B})$

That is, $\quad\left(\lambda^{\mu}\right)^{-}\left(\mathrm{AB}^{-1}\right)=\left(\lambda^{\mu}\right)^{-(}(\mathrm{E}) \Rightarrow\left(\lambda^{\mu}\right)^{-(}(\mathrm{A}) \geq\left(\lambda^{\mu}\right)^{-(}(\mathrm{B})$

Since, $\quad\left(\lambda^{\mu}\right)^{-}\left(\mathrm{BA}^{-1}\right)=\left(\lambda^{\mu}\right)^{-}\left(\left(\mathrm{BA}^{-1}\right)^{-1}\right)$

$$
\begin{aligned}
& =\left(\lambda^{\mu}\right)^{-}\left(\mathrm{AB}^{-1}\right) \\
& =\left(\lambda^{\mu}\right)^{-}(\mathrm{E})
\end{aligned}
$$

$\left(\lambda^{\mu}\right)^{-}\left(\mathrm{BA}^{-1}\right)=\left(\lambda^{\mu}\right)^{-}(\mathrm{E})$

That is, $\quad\left(\lambda^{\mu}\right)^{-}\left(\mathrm{BA}^{-1}\right)=\left(\lambda^{\mu}\right)^{-}(\mathrm{E}) \Rightarrow\left(\lambda^{\mu}\right)^{-}(\mathrm{B}) \geq\left(\lambda^{\mu}\right)^{-}(\mathrm{A})$

Hence, $\quad\left(\lambda^{\mu}\right)^{-}(\mathrm{A})=\left(\lambda^{\mu}\right)^{-}(\mathrm{B})$

Therefore, $\left(\lambda^{\mu}\right)^{-}\left(\mathrm{AB}^{-1}\right)=\left(\lambda^{\mu}\right)^{-}(\mathrm{E}) \Rightarrow\left(\lambda^{\mu}\right)^{-}(\mathrm{A})=\left(\lambda^{\mu}\right)^{-}(\mathrm{B})$, for all A,B in $\vartheta$.

4. Properties of lower level subsets of a bipolar anti l-fuzzy sub $\ell$ - HX group

In this section, We introduce the concept of lower level subsets of a bipolar ani L-fuzzy sub $\ell$ - HX group and discuss some of its properties.

Definition 4.1. Let $\lambda^{\mu}$ be a bipolar anti L-fuzzy sub $\ell$ - HX group of a $\ell$ - HX group $\vartheta$. For any $\langle\alpha, \beta\rangle \in[0,1] \times[-1,0]$, We define the set $\lambda^{\mu}{ }_{\langle\alpha, \beta\rangle}=\left\{A \in \vartheta /\left(\lambda^{\mu}\right)^{+}(A) \leq \alpha\right.$ and $\left(\lambda^{\mu}\right)^{-}$ (B) $\geq \beta\}$ is called the $\left\langle\alpha, \beta>\right.$ lower level subset of $\lambda^{\mu}$.

Theorem 4.1. Let $\lambda^{\mu}$ be a bipolar anti L-fuzzy sub $\ell$ - HX group of a $\ell$ - HX group $\vartheta$ then for $\langle\alpha, \beta\rangle \in[0,1] x[-1,0]$ such that $\left(\lambda^{\mu}\right)^{+}(E) \leq \alpha,\left(\lambda^{\mu}\right)^{-}(E) \geq \beta$ and $\lambda^{\mu}{ }_{\alpha \alpha, \beta}$ is a sub $\ell$ - HX group of $\vartheta$.

Proof: For all $\mathrm{A}, \mathrm{B} \in \lambda^{\mu}{ }_{<\alpha, \beta>}$ we have $\left(\lambda^{\mu}\right)^{+}(\mathrm{A}) \leq \alpha,\left(\lambda^{\mu}\right)^{-}(\mathrm{A}) \geq \beta$ and $\left(\lambda^{\mu}\right)^{+}(\mathrm{B}) \leq \alpha$, 
Bipolar Anti L-Fuzzy Sub $\ell$-HX Group and its Lower Level Sub $\ell$-HX Groups

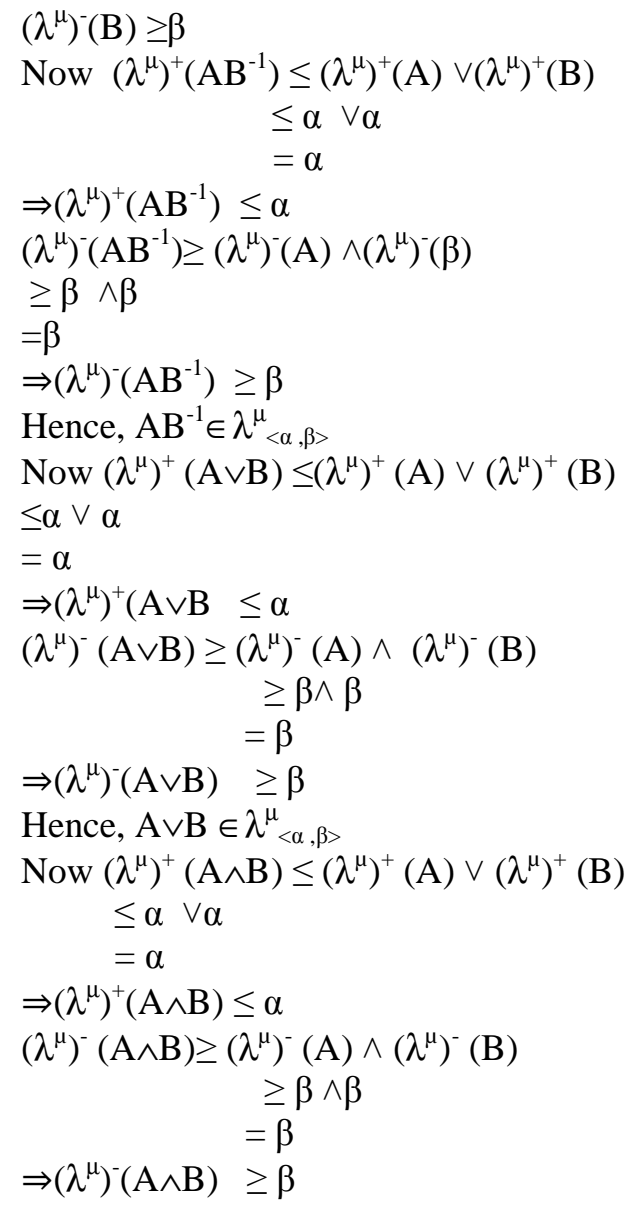

Hence, $\mathrm{A} \wedge \mathrm{B} \in \lambda^{\mu}{ }_{\langle\alpha, \beta>}$

Hence, $\lambda^{\mu}{ }_{\langle\alpha, \beta\rangle}$ is a sub $\ell$ - HX group of $\vartheta$.

Definition 4.2. Let $\lambda^{\mu}$ is a bipolar anti L-fuzzy sub $\ell$ - HX group of a - HX group $\vartheta$. The sub $\ell$ - HX groups $\lambda^{\mu}{ }_{<\alpha, \beta>}$ for $\left\langle\alpha, \beta>\in[0,1] \times[-1,0]\right.$ and $\left(\lambda^{\mu}\right)^{+}(E) \leq \alpha,\left(\lambda^{\mu}\right)^{-}(E) \geq \beta$ are called lower level sub $\ell$ - HX groups of $\lambda^{\mu}$.

Theorem 4.2. Let $\vartheta$ be a $\ell$ - HX group and $\lambda^{\mu}$ be a bipolar anti L-fuzzy subset of $\vartheta$ such that $\lambda^{\mu}{ }_{\langle\alpha, \beta\rangle}$ is a sub $\ell-$ HX group of $\vartheta$ for $\langle\alpha, \beta\rangle \in[0,1] \times[-1,0]$ such that $\left(\lambda^{\mu}\right)^{+}(E) \leq \alpha$, $\left(\lambda^{\mu}\right)^{-}(\mathrm{E}) \geq \beta$.Then $\lambda^{\mu}$ is a bipolar anti L-fuzzy sub $\ell$ - HX group of $\vartheta$.

Proof: Let $\mathrm{A}, \mathrm{B} \in \vartheta$, let $\mathrm{A} \in \lambda^{\mu}{ }_{<\alpha 1,}, \beta 1>\left(\lambda^{\mu}\right)^{+}(\mathrm{A})=\alpha_{1},\left(\lambda^{\mu}\right)^{-}(\mathrm{A})=\beta_{1}$ and $\mathrm{B} \in \lambda^{\mu}{ }_{<\alpha 2}$, ${ }_{\beta 22} \Rightarrow\left(\lambda^{\mu}\right)^{+}(B)=\alpha_{2},\left(\lambda^{\mu}\right)^{-}(B)=\beta_{2}$. Suppose $\lambda^{\mu}{ }_{<\alpha 1, \beta 1}>\lambda^{\mu}{ }_{<\alpha 2, \beta 2>}$ then $A, B \in \lambda^{\mu}{ }_{<\alpha 2, \beta 2>}$ As $\lambda^{\mu}{ }_{<\alpha 2,}$, ${ }_{\beta 2}>$ is a sub $\ell$ - HX group of $\vartheta, \mathrm{AB}^{-1} \in \lambda^{\mu}{ }_{<\alpha 2, \beta 2>}, \mathrm{A} \wedge \mathrm{B} \in \lambda^{\mu}{ }_{<\alpha 2, \beta 2>}$ and $\mathrm{A} \vee \mathrm{B} \in \lambda^{\mu}{ }_{<\alpha 2, \beta 2>}$,

Now $\left(\lambda^{\mu}\right)^{+}\left(\mathrm{AB}^{-1}\right) \leq \alpha_{2}$

$=\alpha_{1} \vee \alpha_{2}$

$=\left(\lambda^{\mu}\right)^{+}(\mathrm{A}) \vee\left(\lambda^{\mu}\right)^{+}(\mathrm{B})$

Hence, $\left(\lambda^{\mu}\right)^{+}\left(\mathrm{AB}^{-1}\right) \leq\left(\lambda^{\mu}\right)^{+}(\mathrm{A}) \vee\left(\lambda^{\mu}\right)^{+}(\mathrm{B})$ 


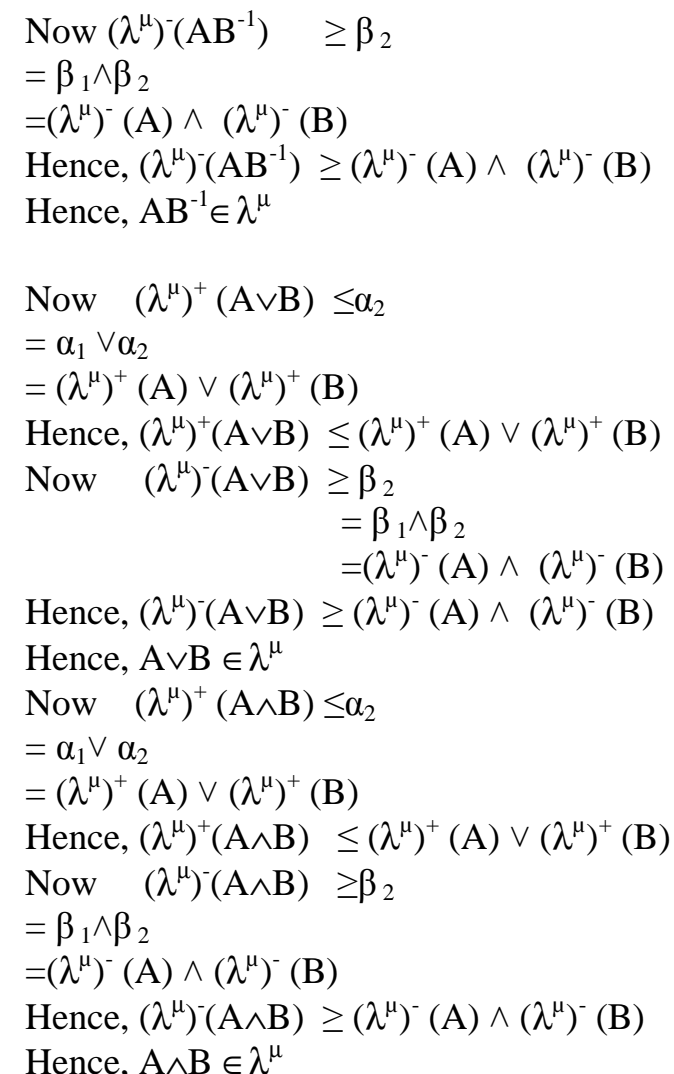

Hence $\lambda^{\mu}$ is a bipolar anti L-fuzzy sub $\ell$ - HX group of $\vartheta$.

Theorem 4.3. Let $\vartheta$ be a $\ell$ - HX group and $\lambda^{\mu}$ be a bipolar anti L-fuzzy sub $\ell$ - HX group of $\vartheta$. If two bipolar lower level sub $\ell$ - HX groups $\lambda^{\mu}{ }_{<\alpha, \gamma>}, \lambda^{\mu}{ }_{<\beta, \delta>}$ with $\alpha<\beta$ and $\delta<\gamma$ of $\lambda^{\mu}$ are equal if and only if there is no $A \in \vartheta$ such that $\alpha<\left(\lambda^{\mu}\right)^{+}(\mathrm{A}) \leq \beta$ and $\delta \leq\left(\lambda^{\mu}\right)^{-}(\mathrm{A})<\gamma$.

Proof: Let $\lambda^{\mu}{ }_{\langle\alpha, \gamma>}=\lambda^{\mu}{ }_{\langle\beta, \delta>}$. Suppose that there exists $A \in \vartheta$ such that $\alpha<\left(\lambda^{\mu}\right)^{+}(\mathrm{A}) \leq \beta$ and $\delta \leq\left(\lambda^{\mu}\right)^{-}(\mathrm{A})<\gamma$. Then $\lambda^{\mu}{ }_{<\alpha, \gamma>} \subset \lambda^{\mu}{ }_{\langle\beta, \delta>}$ since $\mathrm{A} \in \lambda^{\mu}{ }_{\langle\beta, \delta>}$ but not in $\lambda^{\mu}{ }_{<\alpha, \gamma>}$ which contradicts the hypothesis. Hence there exists no $A \in \vartheta$ such that $\alpha<\left(\lambda^{\mu}\right)^{+}(\mathrm{A}) \leq \beta$ and $\delta \leq\left(\lambda^{\mu}\right)^{-}(\mathrm{A})<\gamma$. Conversely, let there be no $A \in \vartheta$ such that $\alpha<\left(\lambda^{\mu}\right)^{+}(\mathrm{A}) \leq \beta$ and $\delta \leq\left(\lambda^{\mu}\right)^{-}(\mathrm{A})<\gamma$. Since $\alpha<$ $\beta$ and $\delta<\gamma$,we have $\lambda^{\mu}{ }_{\langle\alpha, \gamma>} \subset \lambda^{\mu}{ }_{\langle\beta, \delta>}$. Let $A \in \lambda^{\mu}{ }_{\langle\beta, \delta>}$ then $\left(\lambda^{\mu}\right)^{+}(\mathrm{A}) \leq \beta$ and $\left(\lambda^{\mu}\right)^{-}(\mathrm{A}) \geq \delta$. since there exists no $A \in \vartheta$ such that $\alpha<\left(\lambda^{\mu}\right)^{+}(\mathrm{A}) \leq \beta$ and $\delta \leq\left(\lambda^{\mu}\right)^{-}(\mathrm{A})<\gamma$, we have $\left(\lambda^{\mu}\right)^{+}$ (A) $\leq \alpha$ and $\left(\lambda^{\mu}\right)^{-}(\mathrm{A}) \geq \gamma$ which implies $A \in \lambda^{\mu}{ }_{\langle\alpha, \gamma>}$ that is $\lambda_{\langle\beta, \delta}^{\mu} \subset \lambda^{\mu}{ }_{\langle\alpha, \gamma>}$. Hence, $\lambda^{\mu}{ }_{\langle\alpha, \gamma\rangle}=$ $\lambda^{\mu}<\beta, \delta>$.

Theorem 4.4. A L-fuzzy subset $\lambda^{\mu}$ of $\vartheta$ is a bipolar anti L-fuzzy sub $\ell$ - HX group of $\vartheta$ if and only if the lower level subsets $\lambda^{\mu}{ }_{\langle\alpha, \beta>},<\alpha, \beta>\in$ Image $\lambda^{\mu}$ are sub $\ell$ - HX group of $\vartheta$.

Proof. It is clear. 
Bipolar Anti L-Fuzzy Sub $\ell$-HX Group and its Lower Level Sub $\ell$-HX Groups

Theorem 4.5.Any sub $\ell$ - HX group H of a $\ell$ - HX group $\vartheta$ can be realized as a lower level sub $\ell-H X$ group of some bipolar anti L-fuzzy sub $\ell$ - HX group of $\vartheta$.

Proof: Let $\lambda^{\mu}=\left(\left(\lambda^{\mu}\right)^{+},\left(\lambda^{\mu}\right)^{-}\right)$be a bipolar L-fuzzy subset and $\mathrm{A} \in \vartheta$,

Define $\left(\lambda^{\mu}\right)^{+}(A)=\left\{\begin{array}{l}0, \text { if } A \in H \\ \text { if } A \notin H\end{array}\right.$

and

$$
\left(\lambda^{\mu}\right)^{-}(\mathrm{A})=\beta \text {, if } \mathrm{if} \quad \mathrm{A} \in \mathrm{H}
$$

we shall prove $\lambda^{\mu}$ be a bipolar anti L-fuzzy sub $\ell$ - HX group of $\vartheta$.

Let $\mathrm{A}, \mathrm{B} \in \vartheta$.

i) Suppose $A, B \in H$ then $A B \in H, A B^{-1} \in H, A \wedge B \in H$ and $A \vee B \in H$

$\left(\lambda^{\mu}\right)^{+}(\mathrm{A})=\left(\lambda^{\mu}\right)^{+}(\mathrm{B})=0$ and $\left(\lambda^{\mu}\right)^{-}(\mathrm{A})=\left(\lambda^{\mu}\right)^{-}(\mathrm{B})=\beta$

Now $\left(\lambda^{\mu}\right)^{+}\left(\mathrm{AB}^{-1}\right)=0$

$$
\begin{aligned}
& \leq 0 \vee 0 \\
& =\left(\lambda^{\mu}\right)^{+}(\mathrm{A}) \vee\left(\lambda^{\mu}\right)^{+}(\mathrm{B})
\end{aligned}
$$

Hence, $\left(\lambda^{\mu}\right)^{+}\left(\mathrm{AB}^{-1}\right) \leq\left(\lambda^{\mu}\right)^{+}(\mathrm{A}) \vee\left(\lambda^{\mu}\right)^{+}(\mathrm{B})$

Now $\quad\left(\lambda^{\mu}\right)^{-}\left(\mathrm{AB}^{-1}\right)=\beta$

$\geq \beta \wedge \beta$

$=\left(\lambda^{\mu}\right)^{-}(\mathrm{A}) \wedge\left(\lambda^{\mu}\right)^{-}(\mathrm{B})$

Hence, $\left(\lambda^{\mu}\right)^{-}\left(\mathrm{AB}^{-1}\right) \geq\left(\lambda^{\mu}\right)^{-}(\mathrm{A}) \wedge\left(\lambda^{\mu}\right)^{-}(\mathrm{B})$

Now $\left(\lambda^{\mu}\right)^{+}(A \vee B)=0$

$\leq 0 \vee 0$

$=\left(\lambda^{\mu}\right)^{+}(\mathrm{A}) \vee\left(\lambda^{\mu}\right)^{+}(\mathrm{B})$

Hence, $\left(\lambda^{\mu}\right)^{+}(\mathrm{A} \vee \mathrm{B}) \leq\left(\lambda^{\mu}\right)^{+}(\mathrm{A}) \vee\left(\lambda^{\mu}\right)^{+}(\mathrm{B})$

Now $\left(\lambda^{\mu}\right)^{-}(A \vee B)=\beta$

$\geq \beta \wedge \beta$

$=\left(\lambda^{\mu}\right)^{-}(\mathrm{A}) \wedge\left(\lambda^{\mu}\right)^{-}(\mathrm{B})$

Hence, $\left(\lambda^{\mu}\right)^{-}(\mathrm{A} \vee \mathrm{B}) \geq\left(\lambda^{\mu}\right)^{-}(\mathrm{A}) \wedge\left(\lambda^{\mu}\right)^{-}(\mathrm{B})$

Now $\left(\lambda^{\mu}\right)^{+}(A \wedge B)=0$

$\leq 0 \vee 0$

$=\left(\lambda^{\mu}\right)^{+}(\mathrm{A}) \vee\left(\lambda^{\mu}\right)^{+}(\mathrm{B})$

Hence, $\left(\lambda^{\mu}\right)^{+}(\mathrm{A} \wedge \mathrm{B}) \leq\left(\lambda^{\mu}\right)^{+}(\mathrm{A}) \vee\left(\lambda^{\mu}\right)^{+}(\mathrm{B})$

Now $\left(\lambda^{\mu}\right)^{-}(A \wedge B)=\beta$

$\geq \beta \wedge \beta$

$=\left(\lambda^{\mu}\right)^{-}(\mathrm{A}) \wedge\left(\lambda^{\mu}\right)^{-}(\mathrm{B})$

Hence, $\quad\left(\lambda^{\mu}\right)^{-}(\mathrm{A} \wedge \mathrm{B}) \geq\left(\lambda^{\mu}\right)^{-}(\mathrm{A}) \wedge\left(\lambda^{\mu}\right)^{-}(\mathrm{B})$.

ii) Suppose $A \in H, B \notin H$ then $A B \notin H, A B^{-1} \notin H, A \wedge B \in H$ or $A \wedge B \notin H$ and $A \vee B \in H$ or $\mathbf{A} \vee \mathbf{B} \notin \mathbf{H}$.

$\left(\lambda^{\mu}\right)^{+}(\mathrm{A})=0,\left(\lambda^{\mu}\right)^{+}(\mathrm{B})=\alpha$ and $\left(\lambda^{\mu}\right)^{-}(\mathrm{A})=\beta,\left(\lambda^{\mu}\right)^{-}(\mathrm{B})=0$

Define 
R. Muthuraj and G. Santha Meena

- $\left(\lambda^{\mu}\right)^{+}(A \wedge B)=\left\{\begin{array}{l}0, \text { if } A \wedge B \in H \\ \alpha, \text { if } A \wedge B \notin H\end{array}\right.$ and

$$
\begin{aligned}
&\left(\lambda^{\mu}\right)^{-}(A \wedge B)=\left\{\begin{array}{l}
\beta, \text { if } A \wedge B \in H \\
\text {, if } A \wedge B \notin H
\end{array}\right. \\
&\left(\lambda^{\mu}\right)^{+}(A \vee B)=\left\{\begin{array}{l}
0, \text { if } A \vee B \in H \\
\text { if } A \vee B \notin H
\end{array}\right. \text { and } \\
&\left(\lambda^{\mu}\right)^{-}(A \vee B)=\$ \text {, if } A \vee B \in H \\
& \text {, if } A \vee B \notin H
\end{aligned}
$$

\section{Let $A \wedge B \in H$ and $A \vee B \in H$}

Now $\left(\lambda^{\mu}\right)^{+}\left(\mathrm{AB}^{-1}\right)=\alpha$

$$
\begin{aligned}
& \leq 0 \vee \alpha \\
& =\left(\lambda^{\mu}\right)^{+}(\mathrm{A}) \vee\left(\lambda^{\mu}\right)^{+}(\mathrm{B})
\end{aligned}
$$

Hence, $\left(\lambda^{\mu}\right)^{+}\left(\mathrm{AB}^{-1}\right) \leq\left(\lambda^{\mu}\right)^{+}(\mathrm{A}) \vee\left(\lambda^{\mu}\right)^{+}(\mathrm{B})$

Now $\quad\left(\lambda^{\mu}\right)^{-}\left(\mathrm{AB}^{-1}\right)=0$

$$
\begin{aligned}
& \geq \beta \wedge 0 \\
& =\left(\lambda^{\mu}\right)^{-}(\mathrm{A}) \wedge\left(\lambda^{\mu}\right)^{-}(\mathrm{B})
\end{aligned}
$$

Hence, $\left(\lambda^{\mu}\right)^{-}\left(\mathrm{AB}^{-1}\right) \geq\left(\lambda^{\mu}\right)^{-}(\mathrm{A}) \wedge\left(\lambda^{\mu}\right)^{-}(\mathrm{B})$

Now $\left(\lambda^{\mu}\right)^{+}(A \vee B)=0$

$\leq 0 \vee \alpha$

$=\left(\lambda^{\mu}\right)^{+}(\mathrm{A}) \vee\left(\lambda^{\mu}\right)^{+}(\mathrm{B})$

Hence, $\left(\lambda^{\mu}\right)^{+}(\mathrm{A} \vee \mathrm{B}) \leq\left(\lambda^{\mu}\right)^{+}(\mathrm{A}) \vee\left(\lambda^{\mu}\right)^{+}(\mathrm{B})$

Now $\quad\left(\lambda^{\mu}\right)^{-}(A \vee B)=\beta$

$\geq \beta \wedge 0$

$=\left(\lambda^{\mu}\right)^{-}(\mathrm{A}) \wedge\left(\lambda^{\mu}\right)^{-}(\mathrm{B})$

Hence, $\left(\lambda^{\mu}\right)^{-}(A \vee B) \geq\left(\lambda^{\mu}\right)^{-}(A) \wedge\left(\lambda^{\mu}\right)^{-}(B)$

Now $\quad\left(\lambda^{\mu}\right)^{+}(\mathrm{A} \wedge \mathrm{B})=0$

$$
\begin{aligned}
& \leq 0 \vee \alpha \\
& =\left(\lambda^{\mu}\right)^{+}(\mathrm{A}) \vee\left(\lambda^{\mu}\right)^{+}(\mathrm{B})
\end{aligned}
$$

Hence, $\left(\lambda^{\mu}\right)^{+}(\mathrm{A} \wedge \mathrm{B}) \leq\left(\lambda^{\mu}\right)^{+}(\mathrm{A}) \vee\left(\lambda^{\mu}\right)^{+}(\mathrm{B})$

Now $\left(\lambda^{\mu}\right)^{-}(\mathrm{A} \wedge \mathrm{B})=\beta$

$\geq \beta \wedge 0$

$=\left(\lambda^{\mu}\right)^{-}(\mathrm{A}) \wedge\left(\lambda^{\mu}\right)^{-}(\mathrm{B})$

Hence, $\quad\left(\lambda^{\mu}\right)^{-}(\mathrm{A} \wedge \mathrm{B}) \geq\left(\lambda^{\mu}\right)^{-}(\mathrm{A}) \wedge\left(\lambda^{\mu}\right)^{-}(\mathrm{B})$.

Let $A \wedge B \notin H$ and $A \vee B \notin H$

Now $\left(\lambda^{\mu}\right)^{+}\left(\mathrm{AB}^{-1}\right)=\alpha$

$$
\leq 0 \vee \alpha
$$$$
=\left(\lambda^{\mu}\right)^{+}(\mathrm{A}) \vee\left(\lambda^{\mu}\right)^{+}(\mathrm{B})
$$ 
Bipolar Anti L-Fuzzy Sub $\ell$-HX Group and its Lower Level Sub $\ell$-HX Groups

Hence, $\left(\lambda^{\mu}\right)^{+}\left(\mathrm{AB}^{-1}\right) \leq\left(\lambda^{\mu}\right)^{+}(\mathrm{A}) \vee\left(\lambda^{\mu}\right)^{+}(\mathrm{B})$

Now $\left(\lambda^{\mu}\right)^{-}\left(\mathrm{AB}^{-1}\right)=0$

$$
\begin{aligned}
& \geq \beta \wedge 0 \\
& =\left(\lambda^{\mu}\right)^{-}(\mathrm{A}) \wedge\left(\lambda^{\mu}\right)^{-}(\mathrm{B})
\end{aligned}
$$

Hence, $\left(\lambda^{\mu}\right)^{-}\left(\mathrm{AB}^{-1}\right) \geq\left(\lambda^{\mu}\right)^{-}(\mathrm{A}) \wedge\left(\lambda^{\mu}\right)^{-}(\mathrm{B})$

Now $\left(\lambda^{\mu}\right)^{+}(A \vee B)=\alpha$

$$
\leq 0 \vee \alpha
$$

$=\left(\lambda^{\mu}\right)^{+}(\mathrm{A}) \vee\left(\lambda^{\mu}\right)^{+}(\mathrm{B})$.

Hence, $\left(\lambda^{\mu}\right)^{+}(\mathrm{A} \vee \mathrm{B}) \leq\left(\lambda^{\mu}\right)^{+}(\mathrm{A}) \vee\left(\lambda^{\mu}\right)^{+}(\mathrm{B})$

Now $\left(\lambda^{\mu}\right)^{-}(A \vee B)=0$

$$
\begin{aligned}
& \geq \beta \wedge 0 \\
& =\left(\lambda^{\mu}\right)^{-}(\mathrm{A}) \wedge\left(\lambda^{\mu}\right)^{-}(\mathrm{B})
\end{aligned}
$$

Hence, $\quad\left(\lambda^{\mu}\right)^{-}(\mathrm{A} \vee \mathrm{B}) \geq\left(\lambda^{\mu}\right)^{-}(\mathrm{A}) \wedge\left(\lambda^{\mu}\right)^{-}(\mathrm{B})$

Now $\left(\lambda^{\mu}\right)^{+}(A \wedge B)=\alpha$

$$
\begin{aligned}
& \leq 0 \vee \alpha \\
& =\left(\lambda^{\mu}\right)^{+}(\mathrm{A}) \vee\left(\lambda^{\mu}\right)^{+}(\mathrm{B})
\end{aligned}
$$

Hence, $\left(\lambda^{\mu}\right)^{+}(\mathrm{A} \wedge \mathrm{B}) \leq\left(\lambda^{\mu}\right)^{+}(\mathrm{A}) \vee\left(\lambda^{\mu}\right)^{+}(\mathrm{B})$

Now $\left(\lambda^{\mu}\right)^{-}(\mathrm{A} \wedge \mathrm{B})=0$

$\geq \beta \wedge 0$

$=\left(\lambda^{\mu}\right)^{-}(\mathrm{A}) \wedge\left(\lambda^{\mu}\right)^{-}(\mathrm{B})$

Hence, $\left(\lambda^{\mu}\right)^{-}(\mathrm{A} \wedge \mathrm{B}) \geq\left(\lambda^{\mu}\right)^{-}(\mathrm{A}) \wedge\left(\lambda^{\mu}\right)^{-}(\mathrm{B})$.

iii) Suppose $A, B \notin H$ then $A B^{-1} \in H$ or $A B^{-1} \notin H, A \wedge B \notin H$ and $A \vee B \notin H$

$\left(\lambda^{\mu}\right)^{+}(\mathrm{A})=\left(\lambda^{\mu}\right)^{+}(\mathrm{B})=\alpha,\left(\lambda^{\mu}\right)^{-}(\mathrm{A})=\left(\lambda^{\mu}\right)^{-}(\mathrm{B})=0,\left(\lambda^{\mu}\right)^{+}(\mathrm{A} \wedge \mathrm{B})=\left(\lambda^{\mu}\right)^{+}(\mathrm{A} \vee \mathrm{B})=\alpha$ and $\left(\lambda^{\mu}\right)^{-}$ $(\mathrm{A} \wedge \mathrm{B})=\left(\lambda^{\mu}\right)^{-}(\mathrm{A} \vee \mathrm{B})=0$

Define

\section{Let $\mathbf{A B}^{-1} \notin \mathbf{H}$}

$$
\left(\lambda^{\mu}\right)^{+}\left(\mathrm{AB}^{-1}\right)=\left\{\begin{array}{l}
0, \text { if } \mathrm{AB}^{-1} \in \mathrm{H} \\
\text { a } \text {, if } \mathrm{AB}^{-1} \notin \mathrm{H}
\end{array}\right. \text { and }
$$

$$
\left(\lambda^{\mu}\right)^{-}\left(\mathrm{AB}^{-1}\right)=\oint \text {, if } \mathrm{AB}^{-1} \in \mathrm{H}
$$

Now $\left(\lambda^{\mu}\right)^{+}\left(\mathrm{AB}^{-1}\right)=\alpha$

$$
\begin{aligned}
& \leq \alpha \vee \alpha \\
& =\left(\lambda^{\mu}\right)^{+}(\mathrm{A}) \vee\left(\lambda^{\mu}\right)^{+}(\mathrm{B})
\end{aligned}
$$

Hence, $\left(\lambda^{\mu}\right)^{+}\left(\mathrm{AB}^{-1}\right) \leq\left(\lambda^{\mu}\right)^{+}(\mathrm{A}) \vee\left(\lambda^{\mu}\right)^{+}(\mathrm{B})$

Now $\quad\left(\lambda^{\mu}\right)^{-}\left(\mathrm{AB}^{-1}\right)=0$

$$
\begin{aligned}
& \geq 0 \wedge 0 \\
& =\left(\lambda^{\mu}\right)^{-}(\mathrm{A}) \wedge\left(\lambda^{\mu}\right)^{-}(\mathrm{B})
\end{aligned}
$$

Hence, $\left(\lambda^{\mu}\right)^{-}\left(\mathrm{AB}^{-1}\right) \geq\left(\lambda^{\mu}\right)^{-}(\mathrm{A}) \wedge\left(\lambda^{\mu}\right)^{-}(\mathrm{B})$

Now $\left(\lambda^{\mu}\right)^{+}(A \vee B)=\alpha$

$$
\begin{aligned}
& \leq \alpha \vee \alpha \\
& =\left(\lambda^{\mu}\right)^{+}(\mathrm{A}) \vee\left(\lambda^{\mu}\right)^{+}(\mathrm{B})
\end{aligned}
$$

Hence, $\left(\lambda^{\mu}\right)^{+}(\mathrm{A} \vee \mathrm{B}) \leq\left(\lambda^{\mu}\right)^{+}(\mathrm{A}) \vee\left(\lambda^{\mu}\right)^{+}(\mathrm{B})$ 
R. Muthuraj and G. Santha Meena

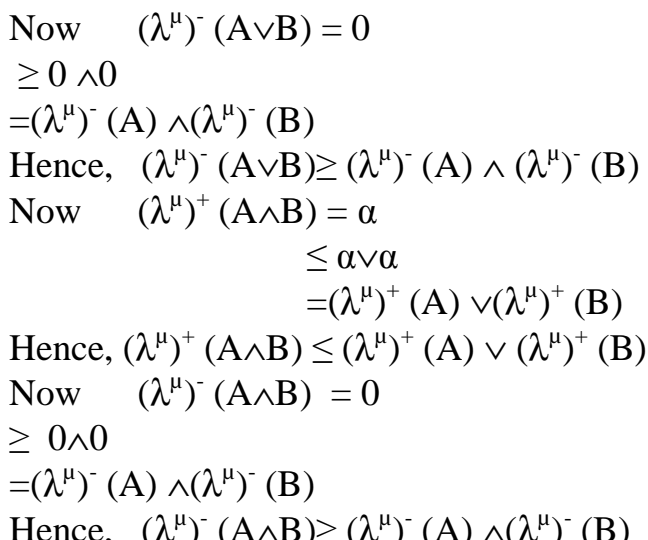

Thus in all cases, $\lambda^{\mu}$ be a bipolar anti L-fuzzy sub $\ell$ - HX group of $\vartheta$. For this bipolar anti L-fuzzy sub $\ell$ - HX group, $\lambda^{\mu}{ }_{\langle\alpha, \beta}=\mathrm{H}$

Remark. As a Consequence of the Theorem 4.3, Theorem 4.4 the lower level sub $\ell$ - HX groups of a bipolar anti L-fuzzy sub $\ell-\mathrm{HX}$ group $\lambda^{\mu}$ of a $\ell$ - HX group $\vartheta$ form a chain. Since $\left(\lambda^{\mu}\right)^{+}(E) \leq\left(\lambda^{\mu}\right)^{+}(A)$ and $\left(\lambda^{\mu}\right)^{-}(E) \geq\left(\lambda^{\mu}\right)^{-}(A)$ for all A in $\vartheta$. Therefore, $\lambda^{\mu}{ }_{<\alpha 0, \beta 0>}$, $\alpha \in[0,1]$ and $\beta \in[-1,0]$. Where $\left(\lambda^{\mu}\right)^{+}(E)=\alpha_{0},\left(\lambda^{\mu}\right)^{-}(E)=\beta_{0}$ is the smallest sub $\ell-H X$ group and we have the chain $\{\mathrm{E}\} \subseteq \lambda^{\mu}{ }_{\langle\alpha 0}, \beta 0>\subseteq \lambda^{\mu}{ }_{\langle\alpha 1}, \beta 1>\subseteq \lambda^{\mu}{ }_{\langle\alpha 2, \beta 2}>\ldots \ldots \ldots \subseteq \lambda^{\mu}{ }_{<\alpha \mathrm{n}, \beta \mathrm{n}\rangle}=$ $\vartheta$, where $\alpha_{0}<\alpha_{1}<\alpha_{2}<\ldots \ldots<\alpha_{\mathrm{n}}$ and $\beta_{0}>\beta_{1}>\beta_{2}>\ldots>\beta_{\mathrm{n}}$.

\section{REFERENCES}

1. P.S.Das, Fuzzy groups and level subgroups, J. Math Anal. Appl., 84 (1981) 264-269.

2. J.A.Goguen, L-Fuzzy sets, J. Math Anal. Appl., 18 (1967) 145-174.

3. K.M.Lee, Bipolar-valued fuzzy sets and their operations, Proc. Int. Conf. on Intelligent Technologies, Bangkok, Thailand ( 2000) 307- 312.

4. Li Hongxing, HX group, BUSEFAL, 33 (1987) $31-37$.

5. L.Chengzhong, Mi Honghai, Li Hongxing, Fuzzy HX group, BUSEFAL41-14 (1989) 97-106.

6. R.Muthuraj and T.Rakeshkumar, Some characterization of L-Fuzzy $\ell$ - HX group, International Journal of Engineering Associates, 38 (2016) 38-41.

7. R.Muthuraj and G.SanthaMeena, Some Characterization of Bipolar L-Fuzzy $\ell-\mathrm{HX}$ group, International Journal of Computational and Applied Mathematics, 1 (2017) 137-155.

8. R.Muthuraj and M.Sridharan, Bipolar fuzzy HX group and its level sub HX groups, International Journal of Mathematical Archive, 5(1) (2014) 230-239.

9. R.Muthuraj and M.Sridharan, Bipolar Anti fuzzy HX group and its lower level sub HX groups, International Journal of Mathematical Archive, 5(1) (2014) 230-239.

10. N.Palaniappan and R.Muthuraj, Anti fuzzy group and Lower level subgroups, Antartica J.Math., 1(1) (2004) 71-76.

11. A.Rosenfeld, Fuzzy groups, J. Math. Anal. Appl., 35 (1971) 512-517.

12. G.S.V.Satyasaibaba, Fuzzy lattice ordered groups, South East Asian Bulletin of Mathematics, 32 (2008) 749-766. 
Bipolar Anti L-Fuzzy Sub $\ell$-HX Group and its Lower Level Sub $\ell$-HX Groups

13. K.Sunderrajan and A.Senthilkumar, Anti L-fuzzy sub $\ell$ group and its lower level sub $\ell$ groups, International Journal of Engineering Science Invention, 2(1) (2013) 21-26.

14. L.A.Zadeh, Fuzzy sets Inform and Control, 8 (1965) 338-365.

15. W.R.Zhang, Bipolar fuzzy sets, Proc. of FUZZ-IEEE (1998) 835-840. 